\title{
A guide to the Nature Index
}

\section{A description of the terminology and methodology used in this supplement, and a guide to the functionality available free online at natureindex.com.}

$\mathrm{T}$ he Nature Index is a database of author affiliations and institutional relationships, used to track contributions to articles published in a group of highly selective science journals that have been chosen by an independent group of active researchers.

Data in the Nature Index are updated monthly, with the most recent 12 months of data made available under a Creative Commons license at natureindex.com.

The Nature Index provides absolute counts of high-quality publication productivity at the institutional and national level, and as such is one indicator of high-quality research output across the globe.

The database is compiled by Nature Publishing Group (NPG) in collaboration with sister company Digital Science.

The current journals tracked by the Nature Index will be reviewed again in 2015 , and from 2016 , coverage will be extended to include the clinical sciences.

\section{NATURE INDEX METRICS}

There are three measures provided by the Nature Index to track affiliation data. The simplest is the article count (AC). A country or institution is given an $\mathrm{AC}$ of 1 for each article that has at least one author from that country or institution. This is the case whether an article has one or a hundred authors, and it means that the same article can contribute to the AC of multiple countries or institutions.

To get a better sense of a country or institution's contribution to an article, and to remove the issue of double-counting of articles, the Nature Index uses the fractional count (FC). FC takes into account the relative contribution of each author to an article.

The total FC available per paper is 1, and this is shared between all authors under the assumption that each contributed equally. For instance, a paper with 10 authors means that each author receives an FC of 0.1. For authors who have worked with joint affiliations, the individual FC is then split equally between each affiliation.

The third measure used is the weighted fractional count (WFC), which applies a weighting to the FC in order to adjust for the over-

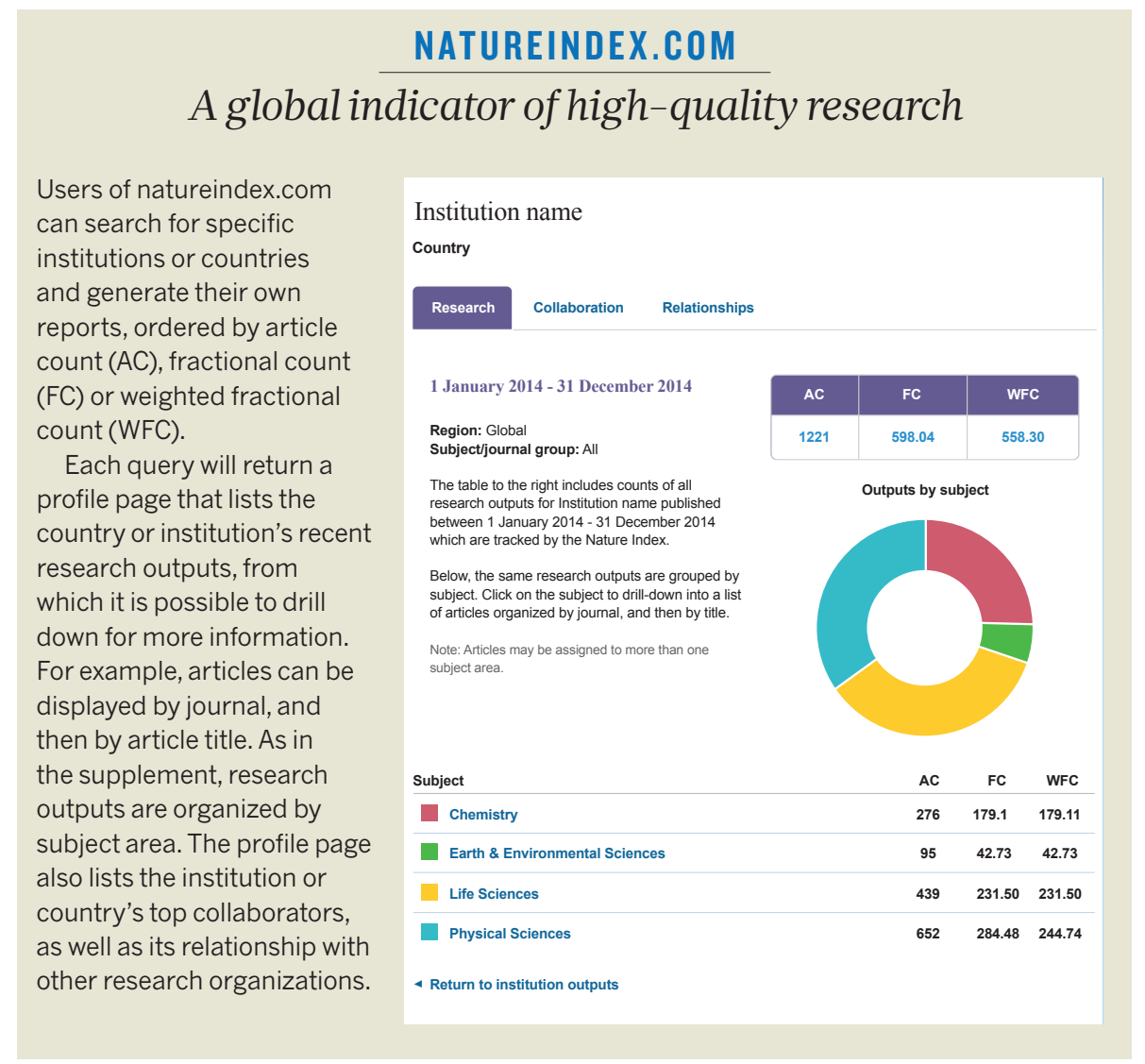

representation of papers from astronomy and astrophysics.

The four journals in these disciplines publish about $50 \%$ of all papers in international journals in this field - approximately fivetimes the equivalent figures for other fields. Therefore, although the data for astronomy and astrophysics are compiled in exactly the same way as for all other disciplines, articles from these journals are assigned one-fifth the weight of other articles (i.e., the FC is multiplied by 0.2 to derive the WFC).

The total FC or WFC for an institution is derived by summing the FC or WFC for individual authors.

The process is similar for countries, although complicated by the fact that some institutions have overseas labs that will be counted towards the host country totals. What's more, there is great variability in the way authors present their affiliations. Every effort is made to count affiliations consistently, and with the background of reasonable assumptions.

For more information on how the affiliation information is processed and counted, please see the frequently asked questions at natureindex.com.

\section{THE SUPPLEMENT}

Nature Index 2015 Asia-Pacific is based on a snapshot of data from natureindex.com, covering articles published between 1 January and 31 December, 2014.

Most analyses within the supplement use the WFC as the primary metric, as it provides a more even basis for comparison, and in determining the relative contribution of each country/institution. 